\title{
SP0125 RENAL HANDLING OF URATE AND GOUT
}

H.-J. Anders. Medizinische Klinik IV, LMU Munich, Muenchen, Germany

Humans, birds, reptiles have (dinosaurs had) a tendency to develop gout because they lack sufficient uricase expression. Elimination of uric acid largely depends on excretion in the urine. A number of factors can limit this process such as genetic variants of uric acid transporters, drug-related inhibition of uric acid excretion, urine $\mathrm{pH}$ or loss of GFR. Visceral gout is a common phenomenon on birds, poultry, and reptiles with renal failure.

Excreting large amounts of uric acid crystals is no problem for birds and reptiles (birds droppings are white for this reason), because urine concentration takes place in the cloacae close to the external orifice. In humans urine concentration takes place in the collecting ducts, which implies that hyperuricosuria imposes a risk for urolithiasis. By contrast, uric acid retention is a common cause for hyperuricemia together with increased purine load from metabolism and diet. Conceptually, a visit is a beer garden is dangerous for two reasons, the intake of purine rich food and drinks (beer) and the intake of fructose-rich soft drinks that blocks certain urate transporters that facilitate urate excretion. A number of well-known triggers for gout relate to renal function. For example, saturnine gout, the gout of the whine-drinking romans or of noblemen of the $17^{\text {th }}$ century related to the cups made of lead or other nephrotoxic metals used at that time that caused chronic kidney disease (CKD)-related hyperuricemia. Also the prevalence of gout and CKD increase both with age. The lecture discusses the role of hyperuricemia and gout in CKD and the role of different therapeutic options in CKD patients.

Disclosure of Interest: None declared

DOI: 10.1136/annrheumdis-2016-eular.6344 